\title{
FIRST PRINCIPLES AND INTEGRATED MODELLING ACHIEVEMENTS TOWARDS TRUSTFUL FUSION POWER PREDICTIONS FOR JET AND ITER
}

\author{
J. GARCIA \\ CEA, IRFM \\ F-13108 Saint-Paul-lez-Durance, France. \\ Email: Jeronimo.garcia@cea.fr \\ R. J. DUMONT, J. JOLY, J. MORALES \\ CEA, IRFM \\ F-13108 Saint-Paul-lez-Durance, France
}

L. GARZOTTI, T.W. BACHE, Y. BARANOV, F.J. CASSON, C. CHALLIS, K. KIROV, J. MAILLOUX, S. SAARELMA

CCFE, Culham Science Centre

Abingdon, Oxon, OX14 3DB, United Kingdom

M. NOCENTE

Dipartimento di Fisica "G. Occhialini", Università di Milano-Bicocca

Piazza della Scienza 3, 20126 Milano, Italy

A. BANON-NAVARRO, T. GOERLER

Max Planck Institute for Plasma Physics

Boltzmannstr. 2, 85748 Garching, Germany

J. CITRIN, A. HO

DIFFER - Dutch Institute for Fundamental Energy Research

TU/e Science Park De Zaale 205612 AJ Eindhoven, The Netherlands

D. GALLART

Barcelona Supercomputing Center (BSC-CNS)

C. Gran Capità 2-4, 08034 Barcelona, Spain

M. MANTSINEN

ICREA-Barcelona Supercomputing Center

C. Gran Capità 2-4, 08034 Barcelona, Spain

AND JET CONTRIBUTORS*

* "See the author list of Overview of the JET preparation for Deuterium-Tritium Operation by E. Joffrin et al. to be published in Nuclear Fusion Special issue: overview and summary reports from the 27th Fusion Energy Conference (Ahmedabad, India, 22-27 October 2018)"

\begin{abstract}
Predictability of burning plasmas is a key issue for designing and building credible future fusion devices. In this context, an important effort of physics understanding and guidance is being carried out in parallel to JET experimental campaigns in $\mathrm{H}$ and $\mathrm{D}$ by performing analyses and modelling towards an improvement of the understanding of DT physics for the optimization of the JET-DT neutron yield and fusion born alpha particle physics. Extrapolations to JET-DT from recent experiments using the maximum power available have been performed including some of the most sophisticated codes and a broad selection of models. There is a general agreement that 11-15MW of fusion power can be expected in DT for the hybrid and baseline scenarios. On the other hand, in high beta, torque and fast ion fraction conditions, isotope effects could be favourable leading to higher fusion yield. It is shown that alpha particles related physics, such as TAE destabilization or fusion power electron heating, could be studied in ITER relevant JET-DT plasmas.
\end{abstract}

\section{INTRODUCTION}

The understanding of the physical mechanisms involved in plasmas composed by a combination of DeuteriumTritium (DT) is an essential activity as such plasmas will be the base for the generation of energy by means of the fusion of D and T nuclei. However, the level of comprehension of burning plasmas is low due to the very few DT 
experiments available for analyses, mostly obtained many years ago in both TFTR [1] and JET [2] tokamaks, and the fact that most of the present-day plasmas are obtained in D. This is an important drawback as significant inherent differences between D and DT plasmas are expected. In particular, heat and particle transport and sources, L-H transition power and pedestal characteristics can significantly change through mechanisms such as the isotope effect, the alpha heating or the helium ash.

Therefore, predictability of burning plasmas is a key issue for the use, designing and building of credible future fusion devices that, unlike experiments in $\mathrm{D}$, will require a specific control and prediction of the neutron rate generation and activation of the Plasma Facing components (PFC). This capability will have to be fully demonstrated for the first time in the framework of the ITER DT operation.

Such predictability requires the integration of several physics aspects which are not fully understood yet even in D plasmas. This is an important challenge due to rather few experimental data in support of any extrapolation activity from D to DT. The future DT campaign scheduled for JET will be an excellent opportunity to overcome that issue by analyzing the physics of DT plasmas and by testing the modelling extrapolation capabilities in view of the future ITER DT plasmas. Towards that goal, an important effort of physics understanding is being carried out in parallel to the JET experimental campaigns by performing analyses and modelling towards an optimization of the JET-DT neutron yield and fusion born alpha particle physics. Whereas the D operation has benefit from this activity, other campaigns with different isotopes, such as $\mathrm{H}$ and $\mathrm{T}$, have also received particular attention by carefully analyzing the interplay between the isotopes and the different aspects of the plasma operation such as turbulence, heating, MHD or energetic particles. This has been done by using different modelling approaches, e.g. from first principle to phenomenological models, with the aim of broadening the scope of the possible results and finding possible discrepancies.

In general, the physics understanding and modelling validation and extrapolation methodology used can be summarized as follows:

- Validation of models on existing D plasmas.

- Verification of a minimum extrapolation capability with existing D plasmas when changing power, Ip and Bt.

- Verification of the extrapolation strategy with future D plasmas. Extension to H and T campaigns.

- Close the 'gap' with respect to DT physics: Validation of models with the first DT campaign at JET (DTE1) and future isotope experiments.

- First-principle modelling supporting the extrapolation strategy

- Transferability to ITER plasmas.

Such program has been already started in previous D campaigns [3] and it has been extended to the H campaign. The future $\mathrm{T}$ campaign has been already started to be prepared in the framework of this plan [4].

This papers report on the main achievements obtained in the framework of this activity and it can be divide as follows. In section 2 transport and turbulence analyses are shown, section 3 is devoted to heat sources, section 4 to DT plasmas extrapolations and analyses, section 5 focuses in particular to alpha particle physics and in section 6 the conclusions will be given.

\section{TRANSPORT AND TURBULENCE}

Transport and turbulence analyses have been performed in D plasmas for the different confinement regimes: baseline, hybrid and scenarios for alpha studies with Internal Transport Barrier (ITB). For such purpose, simulations using the models TGLF [5], QuaLiKiz [6] and CDBM [7] for the heat and particle transport have been carried out.

Regarding the options used for the different models, when applying TGLF the version used is SAT-0 and the experimental rotation is used to calculate the ExB and corresponding parallel velocity gradient (PVG) flow shear. In the case of QuaLiKiz both ExB and PVG are only used in the region $\rho>0.5$ with $\rho$ the normalized toroidal flux. In the region $\rho<0.2$, a fixed turbulence level is added as turbulence from TGLF and QuaLiKiz tend to be too small. These options are identical throughout the paper. In addition to turbulence transport, neoclassical transport has been also simulated by using the NCLASS model [8]. The approaches of CRONOS [9] and JINTRAC [10] regarding particle transport are different, whereas CRONOS is an electron particle solver, JINTRAC is an ion particle solver. In both cases, the averaged measured Zeff and total radiation are constraints for the ion content, 
in particular the impurity composition for each discharge analyzed in this paper. In the case of CRONOS, the impurity profiles are homothetic to the electron density profile whereas for JINTRAC the transport is selfconsistent calculated and the edge values adjusted in order to fulfil the constraints. In general, both codes agree on particle transport as it has been shown in dedicated benchmarks [11].

Globally such models are in reasonable agreement with experimental data although some caveats are found. In the case of TGLF, previous analyses performed for the hybrid scenario with the CRONOS suite of codes found a good agreement with experimental data taken from a JET power scan [3,12] however, such results were very sensitive to the ExB shearing stabilization, which leads to an overestimation of the thermal energy confinement if the experimental rotation is taken into account. For the hybrid regime, an optimum neutron rate can be found depending on the interplay between the electron density and the beam penetration.

These analyses have been extended to the baseline discharge \#92436 [13] with a record neutron rate $\mathrm{R}_{\mathrm{DD}}=2.8 \times 1016 \mathrm{~s}-1$ with both CRONOS and JINTRAC by using different boundary conditions approach. In the case of CRONOS simulations, the pedestal top temperature is calculated following Cordey scaling [14] after matching the pedestal top density by reducing the particle transport to the ion neoclassical thermal transport and adjusting the particle sources. The pedestal width is taken from experiment. This procedure has given good results in the past for the JET C-wall plasmas [15]. In the case of JINTRAC simulations, the boundary condition is fixed to experimental values at normalized toroidal flux radius $\rho=0.8$ but, unlike in the TGLF simulation with CRONOS, where experimental rotation is used, the momentum transport equation is also solved.


FIG. 1. Comparison between simulated and experimental temperature (electron, Te, and ion, Ti) and electron density $\left(\mathrm{n}_{\mathrm{e}}\right)$ profiles for the discharge 92436 at $\mathrm{t}=50.4 \mathrm{~s}$ simulated with CRONOS and TGLF taking the experimental rotation profile. Comparison between simulated and experimental temperature (electron and ion), electron density and rotation profiles for the discharge 92436 at $\mathrm{t}=50.4 \mathrm{~s}$ simulated with JINTRAC and Qualikiz. High Resolution Thomsom Scatering (HRTS) is used for ne and Te, Charge Exchange (CX) for Ti and the toroidal rotation. The profiles are shifted in order to have $\mathrm{Te}=100 \mathrm{eV}$ at the separatrix. 
In Fig. 1, the comparison between the simulated electron and ion temperature profiles and the electron density profile with experimental data is shown for the CRONOS simulation using TGLF. The quasi-linear model QuaLiKiz was also applied to this shot in the framework of the JINTRAC suite of codes. As shown in Fig. 1, the agreement between the electron temperature for both models and the rotation profile for QuaLiKiz with experimental data is quite good, however the ion temperature profile is slightly underestimated and the density peaking overestimated especially for QuaLiKiz in the outer part of the plasma. At least for TGLF, the results tend to be very sensitive to the ExB shearing as it happened with the hybrid scenario [3]. This extreme sensitivity can mask other physical mechanism also playing a role in the Ion Temperature Gradient (ITG) turbulence stabilization, such as non-linear electromagnetic and fast ions effects [16,17], which are not included in this model.

The advanced scenarios shots with ITB, developed in view of for studying fusion born alpha particles, in particular $\alpha$-driven toroidal Alfvén Eigen modes (TAEs) [18], have been also analyzed with the model CDBM, which is known to well describe ITB physics both for JET and JT-60U tokamaks [19]. In Fig. 2 is shown the comparison between the experimental and simulated electron and ion temperature and density profiles for the discharge 92054 at $\mathrm{t}=46.4 \mathrm{~s}$. These simulations have been performed with the CRONOS code. Taking boundary conditions from experimental data at $\rho=0.9$. The agreement between the model and the experimental data is quite remarkable for both the electron temperature and density profiles whereas for the ion temperature it is slightly underestimated in the inner core. The reason might be, as pointed out in reference [19], that no ExB shearing model has been applied for this simulation. In any case, this model can give a conservative prediction for ITB plasmas as also pointed out in [19].

Modelling of $\mathrm{H}$ plasmas has been also carried out in order to assess the validity of previous models to reproduce transport and turbulence with different isotopes. In particular, for heat and particle transport, quasi-linear models tend to deviate more in $\mathrm{H}$ than in D. An example is shown in Fig. 3 and Fig. 4, where the electron and ion temperature and the electron density profiles of the discharges $91450 \mathrm{in} \mathrm{H}$ and 89723 in $\mathrm{D}$ are compared to TGLF simulations with JINTRAC. In this case, it is assumed $\mathrm{Ti}=\mathrm{Te}$ as there are not Ti measurements. Whereas the simulations are in reasonable agreement for the discharge in $\mathrm{D}$, the confinement for the $\mathrm{H}$ discharge is overestimated, in particular the density, which is very similar for both shots [20], is significantly overestimated in $\mathrm{H}$ with TGLF.


FIG 2. Comparison between simulated and experimental temperature (electron and ion) and electron density profiles for the discharge 92054 at $\mathrm{t}=46.4 \mathrm{~s}$ simulated with CRONOS and CDBM. Boundary conditions from experiment are applied at $\rho=0.9$. 

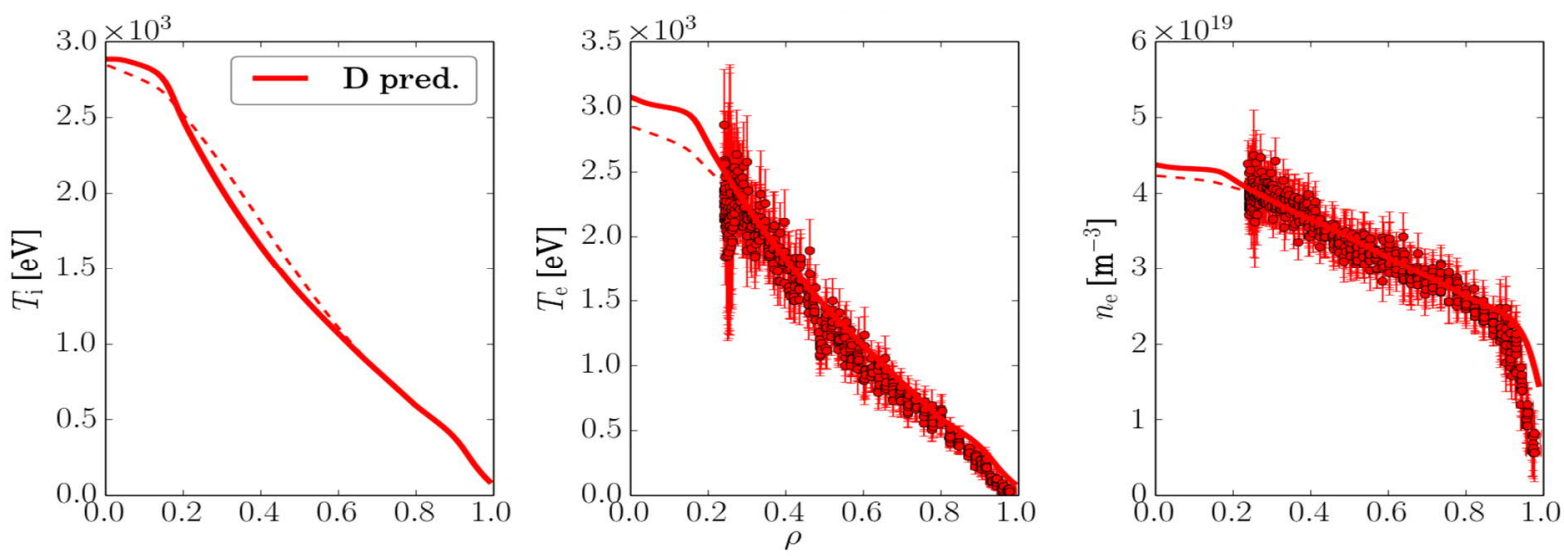

FIG.3. Comparison between simulated (solid) with TGLF and experimental (dashed) electron and ion temperatures and electron density profiles for the discharge 89723 in D in L-mode. Boundary conditions from experiment are applied at $\rho=0.8$
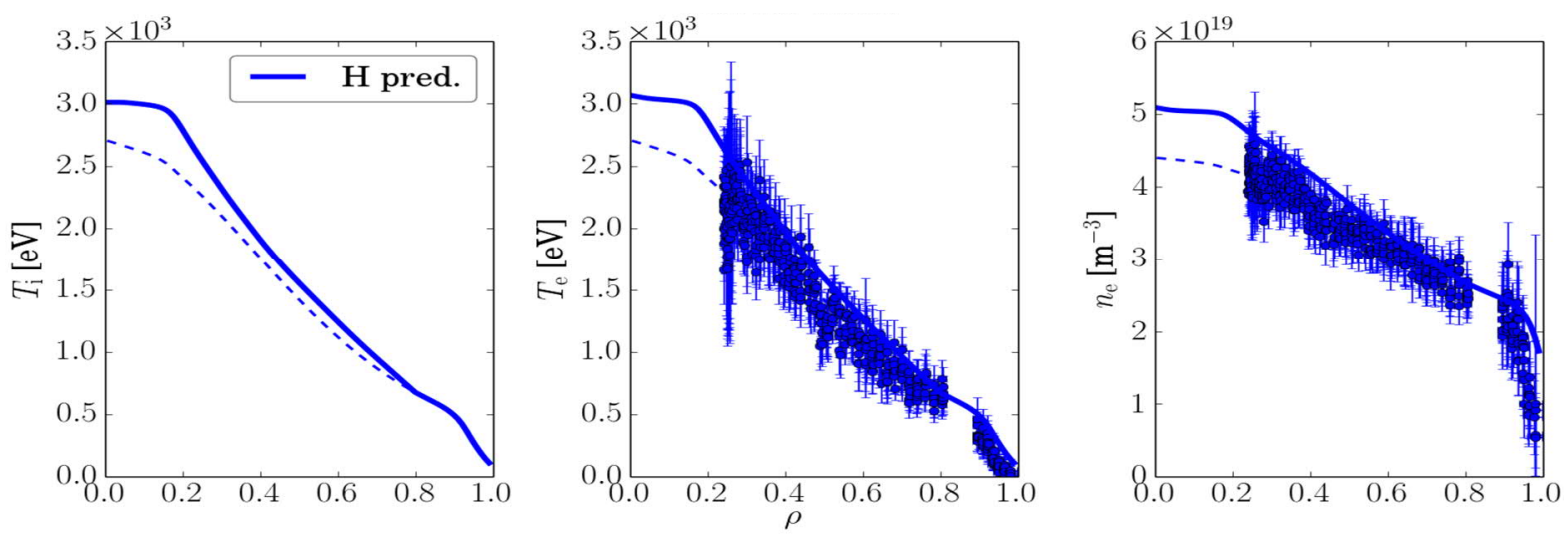

FIG.4. Comparison between simulated (solid) with TGLF and experimental electron and ion temperatures and electron density profiles for the discharge 91450 in $\mathrm{H}$ in L-mode. Boundary conditions from experiment are applied at $\rho=0.8$

Therefore, the comparison of those models against gyrokinetic simulations has been started which has led to a significant improved understanding of the isotope effect. The understading of the reasons for the isotope effect has been extensevaly analysed [21,22]. Gyrokinetic simulations performed with the GENE [23] code show that the fast ion fraction, the ExB shearing rate, the electromagnetic effects and zonal flows, can lead to deviations from the expected GyroBohm (GB) scaling [24] in ITG dominated plasmas. Extra analyses have been performed for the L-mode discharge 91450 in $\mathrm{H}$ by artificially changing the mass from $\mathrm{H}$ to D. As shown in Fig. 5, the ratio $\mathrm{Q}_{\mathrm{D}} / \mathrm{Q}_{\mathrm{H}}$ significantly deviates from $\mathrm{Q}_{\mathrm{D}} / \mathrm{Q}_{\mathrm{H}} \sim 1.4$ which is expected from the GyroBohm scaling of the form $Q_{i} \sim m_{i}{ }^{1 / 2}$. However, the GyroBohm scaling is progressively obtained when removing effects such as external ExB shearing, collisionality or trapped electrons. In particular, the collisionality dependence on the isotope effect seems to depend on the turbulence regime. As shown in Fig.5, the $Q_{D} / Q_{H}$ deviation from GyroBohm scaling depends on whether the plasma is in a ITG+TEM turbulence regime (for which the dependence is weak) or pure Trapped Electron Mode (TEM) (for which the dependence is strong). Extra analyses are being performed for disentangling the difference isotope dependences in core transport at JET [25]. 


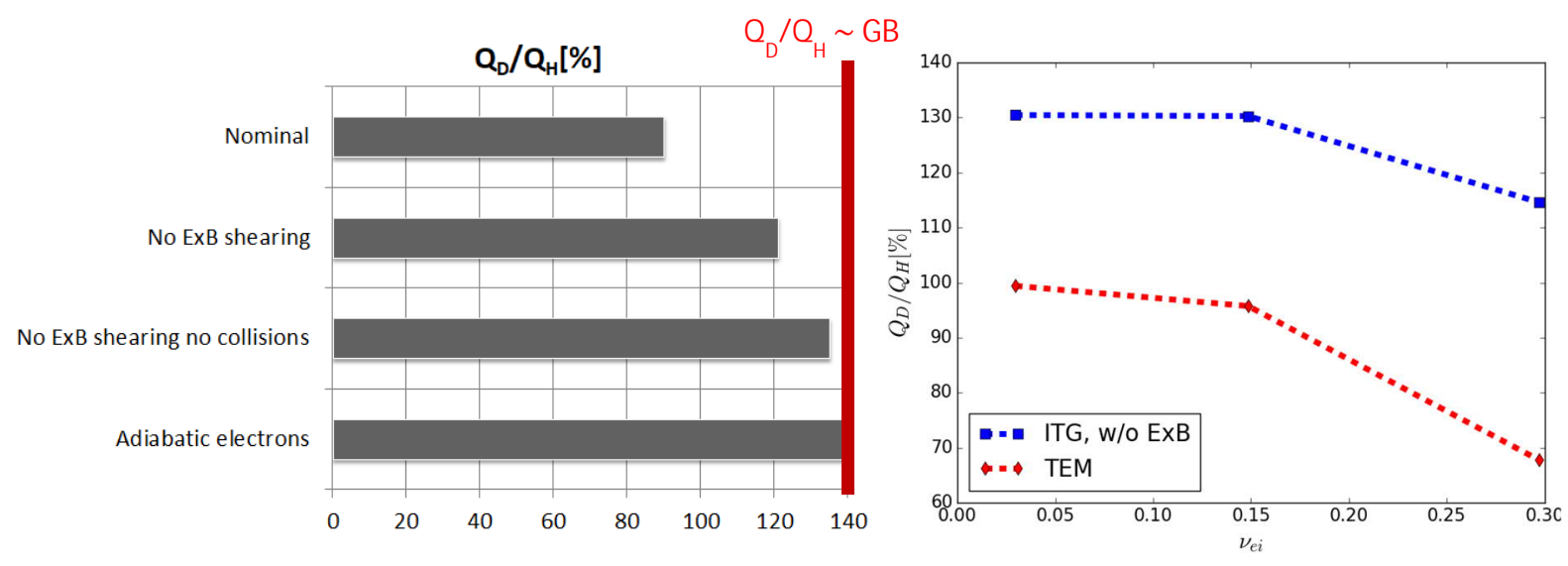

FIG.5. Ratio of the ion heat flux in deuterium and hydrogen $Q_{D} / Q_{H}$ obtained with GENE from the discharge 91450 . The vertical red line denotes the GyroBohm scaling (Left) Dependence of the ratio $Q_{D} / Q_{H}$ on collisionality for ITG vs TEM plasmas based on the discharge 91450 . The transition between the different turbulence regimes was obtained by setting the different species gradients to zero. (Right)

\section{HEAT SOURCES AND NEUTRON RATE GENERATION}


FIG. 6. Comparison between the experimental and simulated neutron rate for the discharge 92436 with TRANSP+TORIC in the case with ICRH power (red), without ICRH power (blue) and without ICRH but with kinetic profiles as in the reference case with PICRH=5.5MW (magenta) (left) the fast ion distribution function (solid lines) and Maxwellian bulk distribution function (dashed lines) with 0 and 5.5MW of ICRH (right)

The neutron rate predictability in DT plasmas is important in order to avoid strong deviations from the expected first wall activation. This is essential for the assessment of the lifetime of any tokamak device producing significant fusion energy, such as ITER. Therefore, a significant amount of work has been devoted to analyze whether the DD reaction neutron rate can be predicted at JET and how to extrapolate such rates to DT plasmas. One key point observed in the plasmas analyzed, which combine heating by Neutral Beam Injection (NBI) and Ion Cyclotron Ressonant Heating (ICRH) waves with a $\mathrm{H}$ minority scheme, is the need to model the damping of the ICRH wave on the fast D obtained from the NBI heating by means of the 2nd harmonic. In particular, for the hybrid scenarios, such interplay can significantly increase the tail of fast ions and boost the beam-target neutron rate generation up to 15-25\% [26]. In the case of the baseline discharge \#92436 the same type of analysis has been carried out with the code package TRANSP and TORIC.

Calculated DD neutron yields were analyzed [27] with the intention of separating the impact of sheer synergistic effects due to changes in fast ions distribution function from supplementary effects accompanying the application of ICRH power, namely changes in Te and Ti. This is done by predictive runs with and without ICRH power 
followed by three TRANSP runs; one with ICRH power, one without ICRH power and kinetic profiles from $\mathrm{PICRH}=0$ predictive run and the third run was without ICRH power, but kinetic profiles were from $\mathrm{PICRH}=5.5 \mathrm{MW}$ predictive run. The sheer synergistic effects are weaker, about 5\%, compared to supplementary effects as shown in Fig. 6 (left) red and magenta lines. If supplementary effects related to changes in Te and Ti with ICRH power are accounted for decrease of fusion performance is significant, about $40 \%$ as shown in Fig. 6. Power scans show that higher ICRH power will pull larger tail in FI DF, Fig. 6 (right), which will further benefit DD reaction rates hence sheer synergistic effects. Calculations show [27] that hybrid plasma benefits more from sheer synergistic effects and this is partly due to the fact that the beam penetration is baseline regimes is worse than in the hybrid ones and therefore the number of NBI ions at the resonance ICRH layer is lower

\section{DT PLASMAS ANALYSES AND FUSION POWER EXTRAPOLATIONS}

Extrapolation to DT from DD plasmas have been performed with several models with the aim of understating the physics mechanisms leading to potential differences between the two ion compositions and guiding the development of $\mathrm{D}$ scenarios in order to maximize the impact of fusion born alpha particles and the fusion power generation. In particular, the dependence on the density, input power, toroidal current and magnetic field have been analyzed. In general such extrapolations have to be performed including models for core turbulence and pedestal top pressure which can ensure a low power degradation at high NBI power due to a strong link between core and pedestal regions [17]. This is especially important for the predictions of low gas and high beta plasmas, which is often the case for hybrid scenarios.

Simulations performed with the CRONOS code and the model TGLF for core turbulence and cordey scaling for the pedestal energy, which includes a mass dependence, have been performed for the extrapolation to DT of the D hybrid discharge 86614 [3] to 40MW of input power. A significant isotope effect was found, linked to the stronger effect of ExB shearing with increasing mass, which leads to a higher core ion temperature for the DT case. However, such improvement in confinement can be partially counteracted by the fact that the core NBI heating and fueling is worse in DT due to the high beam mass. Therefore, for the hybrid scenario, a good density control is required in order to search for an optimum of the fusion power generation but this is particularly difficult as in H-mode the density is mainly determined by the density at the top of the pedestal which in turn depends on Ip.

Extrapolations to $40 \mathrm{MW}$ of the hybrid discharge 92398 [28] with a record neutron rate $\mathrm{R}_{\mathrm{DD}}=2.7 \times 10^{16} \mathrm{~s}^{-1}$ have been also performed with JINTRAC and the turbulent transport model QuaLiKiz (in this case the code NEO [29] is used for the neoclassical transport). In this case, the pedestal is kept fixed as a conservative assumption. An isotope effect is also found leading to higher confinement in DT than DD both for particle and heat transport as shown in Fig. 7 for the case of the temperatures. In this case, since the Electron Transport Gradient (ETG) scales are included in the simulations, the electron temperature does not change with the isotope.

As pointed out in reference [24], the strength of the isotope effect found depends on the plasma conditions. In the case of the discharge 92436, extrapolated as well with the CRONOS code with the same procedure to Ip=3.5MA with 40MW of input power, the differences between DD and DT plasmas are less important. In this case, plasma regions with better and worse confinement in DT with respect to DD alternate as exemplified for the ion thermal pressure in Fig. 7.

Additional extrapolations to DT have been performed with the model Bohm-GyroBohm describing core turbulence and EUROped in a self-consistent interplay [30]. Neglecting any effects from isotopes, the projection using the self-consistent core-pedestal model for the fusion power for a DT JET plasma with toroidal current Ip = 3.8 MA, toroidal magnetic field $\mathrm{Bt}=3.46 \mathrm{~T}$ and $\mathrm{P}_{\text {in }}=40 \mathrm{MW}$ is found to be 11 and $13 \mathrm{MW}$ for the $\mathrm{Ip}=2.5 \mathrm{MA}$, $\mathrm{Bt}=2.9 \mathrm{~T}$ and $\mathrm{P}_{\text {in }}=38 \mathrm{MW}$. The main reason for the better fusion performance of the low current case is that it accesses the part of the stability, where the pedestal limiting mode is an intermediate-n Peeling Ballooning Mode (PBM), while the high current case is limited by high-n ballooning modes. The former has a higher stability limit for a given pressure gradient. 

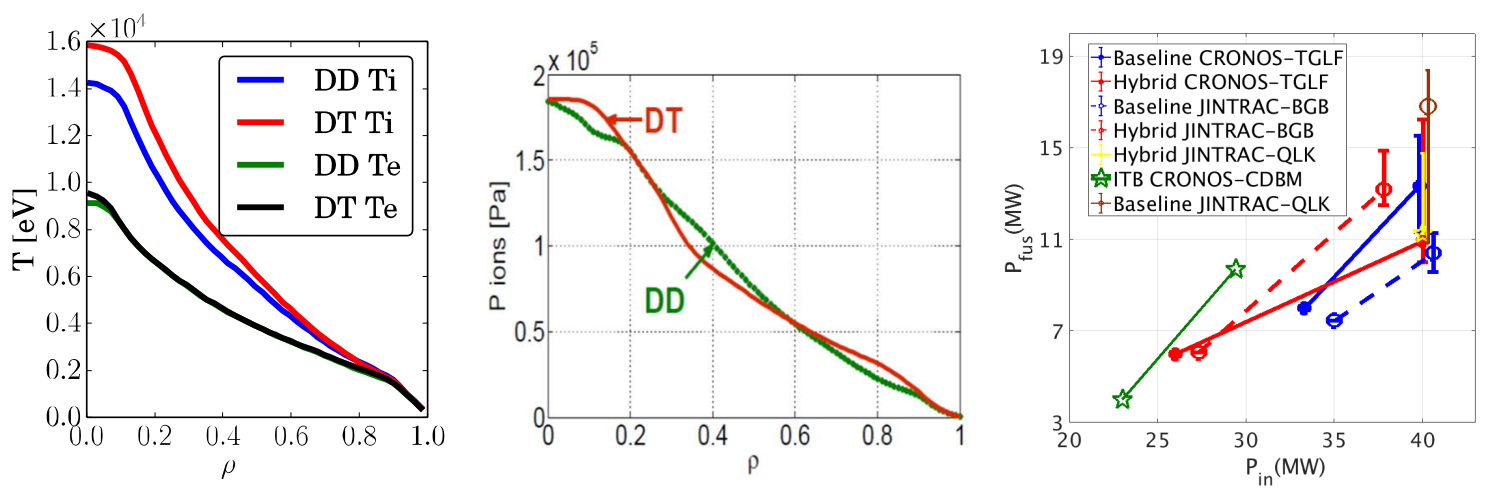

FIG. 7. Comparison between DD and DT for the extrapolation performed to maximum power in DT with JINTRAC and qualikiz for the discharge 92398 (left). Comparison between DD and DT for the extrapolation performed to maximum power in DT with CRONOS and TGLF for the discharge 92436 (center) Extrapolations performed to maximum power in DT with JINTRAC and CRONOS for the hybrid, baseline and advanced scenario routes. Error bars account for: bootstrap current models, isotope effects and total current (right)

A summary of the fusion power expected for the three scenarios analyzed in this paper and different input power is shown in Fig. 7. Interestingly, in spite of the different modeling approaches, there is a general agreement, as shown in Fig. 7, that 11-15MW of fusion power can be expected in DT for the hybrid and baseline scenarios if no strong favorable isotope effects are considered. On the other hand, in high beta, torque, fast ion fraction and Ti/Te conditions, as it is usually the case in hybrid scenarios, isotope effects could be favorable [31] leading to even higher fusion yield. This is in line with the fusion power aimed for such campaign.

It is worth pointing out that the edge conditions, in terms of pedestal top temperature and density and separatrix density, are difficult to predict due to the lack of well stablished simplified models able to account for the physics in that particular region. Some of the attempts used in this paper, as Cordey scaling or EPED, have given good results in some plasma conditions at JET however their validity in the target plasmas aimed in the DT campaign is unknown. A significant effort towards the creation of models valid for the Iter Like Wall (ILW) and for different isotopes is being carried out at JET. This effort will give the opportunity to perform more reliable integrated modelling including core and edge plasma regions and their interplay.
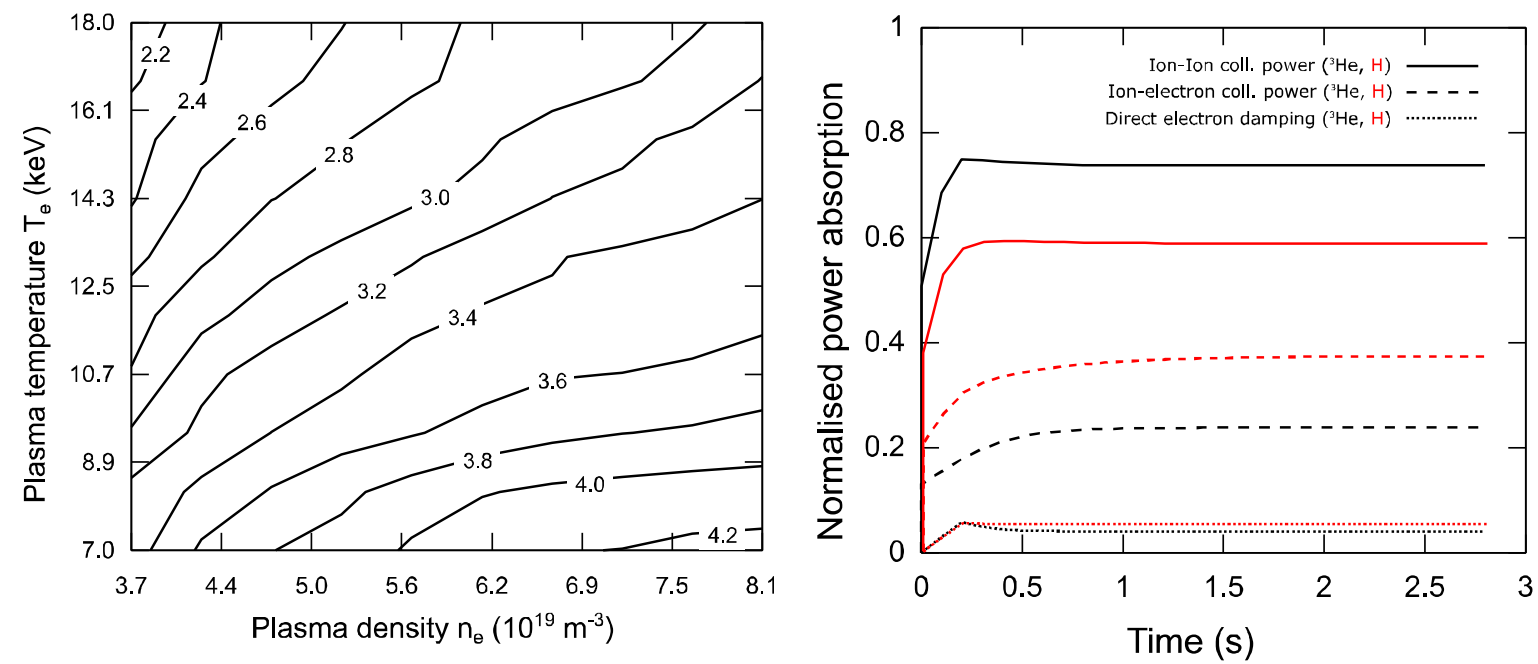

FIG. 8. Difference in total collisional power to the bulk ions (in MW) for a ${ }^{3} \mathrm{He}$ compared to $\mathrm{H}$ minority scheme (left) Ionion collisional heating (solid), ion-electron collisional heating (dashed) and direct electron heating (dotted) for ne $=6 . e 10^{19}$ and $\mathrm{Te}=\mathrm{Ti}=8.9 \mathrm{keV}$ (right)

An important aspect in these simulations is the efficiency of the ICRH system to heat the thermal ions in order to increase the thermal fusion reactions and to prevent $\mathrm{W}$ accumulation. Several ion schemes, as $\mathrm{H}$ or ${ }^{3} \mathrm{He}$ minorities or the three ion schemes [32] are being tested at JET for that purpose. The efficiency of the $\mathrm{H}$ and ${ }^{3} \mathrm{He}$ schemes in DT plasmas has been analyzed with the PION code by performing simulations in a 50\%/50\% DT fuel ion 
mixture under otherwise the same conditions than in the hybrid discharge 86614 . The minority concentrations for $\mathrm{H}$ and ${ }^{3} \mathrm{He}$ are maintained constant at $5 \%$ over $\mathrm{D}$ and $\mathrm{T}$ densities. A scan on Te and $\mathrm{n}_{\mathrm{e}}$ is performed in order to show the different power absorption by the ions. As shown in Fig. 8, the absorbed power is higher with the ${ }^{3} \mathrm{He}$ minority scheme and it increases with respect the $\mathrm{H}$ minority scheme at higher $\mathrm{n}_{\mathrm{e}}$ and lower Te. The direct electron damping remains very similar as also shown for one point of the scan in Fig.8. This shows the potential of the ${ }^{3} \mathrm{He}$ scheme to boost the thermal neutron rate production.

\section{ALPHA PARTICLE PHYSICS}
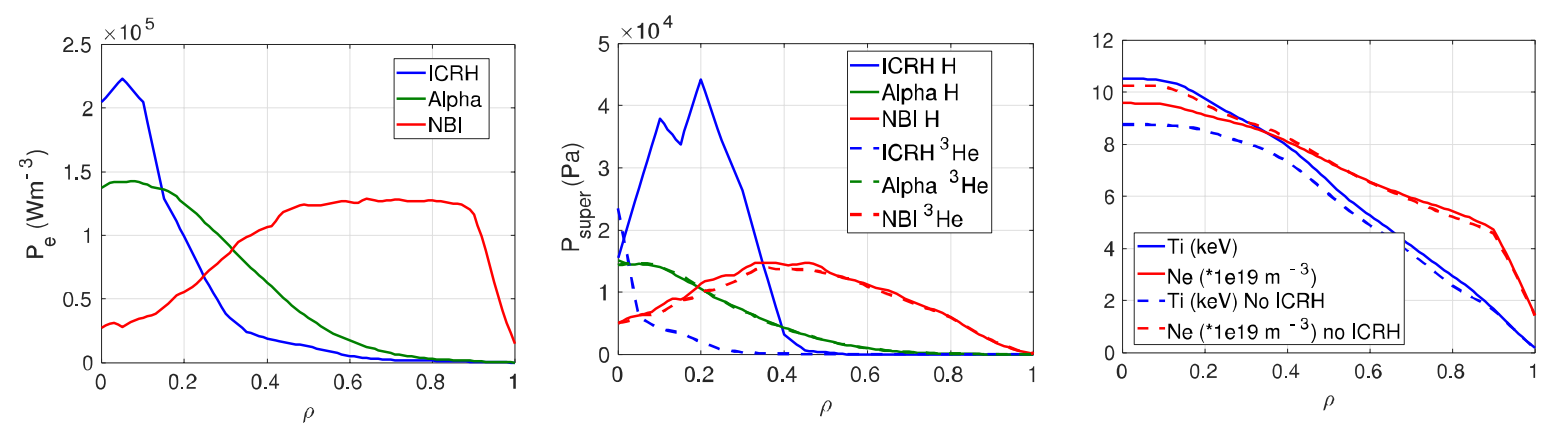

FIG. 9. NBI, ICRH and alpha electron power density heating for the extrapolation of the discharge 92436 to DT with $\mathrm{Ip}=3.5 \mathrm{MA}, 40 \mathrm{MW}$ (left) and the corresponding ICRH, NBI and alpha fast ion pressure for the same case with $\mathrm{H}$ and ${ }^{3} \mathrm{He}$ ICRH schemes (center) Ion temperature and electron density profiles with ICRH H scheme and $0.5 \mathrm{~s}$ after removing the ICRH power (right)

The scenarios obtained indicate that interesting physics could be analyzed in JET-DT plasmas. For the first time in ITER relevant conditions, i.e. the fast ion slowing down time similar to the energy confinement time, the alpha heating to the electrons can be significant, as illustrated in Fig. 9 for the extrapolation made with CRONOS at $\mathrm{Ip}=3.5 \mathrm{MA}$ and Pin=40MW shown in Fig. 7 (with the code PION [33] for the calculation for the ICRH characteristics). This is because most of the electron heating from the NBI is off-axis due to the poor penetration of the beams at this density. However, such heating is in direct competition with the ICRH heating as also shown in Fig. 9. This is a drawback for the observation of alpha heating effects. This feature can also be observed by comparing the fast ion pressure from the different heating mechanism as shown in Fig. 9. Clearly, the fast ions pressure from the ICRH is dominant in the core over the alpha contribution. Several strategies have been considered in order to favor the observation of a clear impact of fusion born alphas on the plasmas considered. Regarding the heating, ICRH could be removed for some determined time in order to leave the alpha heating as the main central electron heating. When this is done with CRONOS, after 0.5s, i.e. about two slowing down times, the fusion power drops by $13 \%$ which means that the fusion power could be still quite significant in the core even without ICRH. However, the removal of ICRH could also impact other plasmas aspects which could be more deleterious, such us the tungsten transport to the core. As shown in Fig. 9, when the ICRH is removed, there is a significant drop of the ion temperature (and its gradient) and an increase of the electron density peaking in the inner core region which favors the core inward transport of tungsten by the neoclassical effects [34].

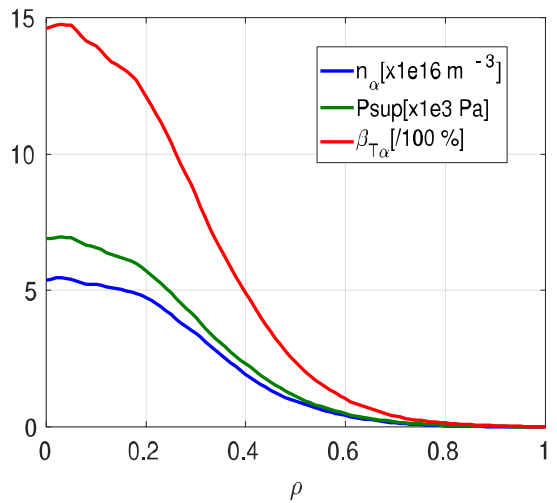

FIG. 10. Fast ion alpha density, pressure and $\beta_{\mathrm{T}}$ for the extrapolation to DT and $31 \mathrm{MW}$ of input power of the discharge 92054
In order to generate fewer ICRH fast ions one possible way is to change the ICRH scheme from $\mathrm{H}$ to ${ }^{3} \mathrm{He}$ minority, which has been shown to increase the ion heating in previous DT plasma extrapolations studies with the PION code [26]. Such possibility has been also analyzed with the CRONOS code, as shown in Fig. 8. In the case of ${ }^{3} \mathrm{He}$ the fusion power is quite similar to the $\mathrm{H}$ case however the fast ion generation is much lower. This possibility will be explored in future JET campaigns.

The possibility of destabilizing TAEs by means of fusion born alpha particles has been also analyzed for the discharge 92054 by performing an extrapolation to $31 \mathrm{MW}$ in DT with the model CDBM. As observed in Fig. 10, $\beta_{\mathrm{T} \alpha}$ can reach $\beta_{\mathrm{T} \alpha} \sim 0.15 \%$ in the core, which is slightly higher than the one obtained by using 
TRANSP extrapolations [18]. Such value is clearly larger than the ones achieved in successful TFTR experiments [35].

\section{CONCLUSIONS}

A strong modelling program in support of the future JET-DT campaign has been started with the aim of guiding the development of the future JET experimental campaigns towards the objectives of maximizing the effects of alpha particle generation on plasmas and minimizing the risk of strong deviations from expected JET-DT results. For that purpose models for transport, turbulence, heating and neutron rate have been compared to $\mathrm{D}$ and $\mathrm{H}$ plasmas and the extrapolability to JET-DT and ITER has been also studied.

In general the models can reproduce D plasmas with some caveats. Quasi-linear models for heat and particle transport can reasonably reproduce temperatures and densities however they also neglect some important physical mechanism know to supress ITG transport, such as non-linear electromagnetic and fast ion effects or multiscale interactions. This might be the reason for the less reliable simulations obtained for $\mathrm{H}$, for which gyrokinetic simulations show that mechanisms such as zonal flows and collisionality also play a role to explain deviations from GyroBohm expectations. Additionally, the strength of such mechanisms on the so called isotope effect could depend on the turbulence regime (ITG vs TEM) which shows the richness of the problem. Further studies will be performed towards the full understanding of those effects. Additionally, the edge conditions, such as the pedestal height and width, are less understood and therefore either fixed values or scalings from C-wall are applied. In spite of the fact that the results tend to be acceptable, the development of the codes towards truly core-edge simulations, with models accounting for that plasma region in the ILW, is required.

Regarding neutron rate predictions, some differences between experimental and predicted neutron yield are systematically obtained without any clear trend. However such differences are less important for plasmas heated with NBI and ICRH if the synergy between both heating mechanisms, through the second harmonic of D in H minority plasmas, is taken into account. On the one hand, such effects account for an increase in neutron rate up to $20 \%$ in hybrid scenarios in D plasmas. In DT plasmas, the interplay can be lower due to the different DT fusion cross section compared to DD plasmas.

Extrapolations to DT have been performed from DD plasmas assuming the maximum power availability at JET. Whereas for the baseline scenario, it is always found that the maximum fusion power generated is obtained at the maximum current possible, $4 \mathrm{MA}$, for the hybrid scenario the necessity of central NBI heating leads to the existence of an optimum in terms of density, which highly depends on the toroidal current used. Interestingly, all the predictions performed for the baseline and the hybrid scenarios lead to a fusion power of 11-15MW, regardless the modelling approach used. Even more interesting is the fact that when using quasi-linear models for the heat transport a significant isotope effect, which improves the thermal confinement with respect to DD, is found in DT simulations. Such improvement in confinement is accompanied by an increasing of the ion temperature and an increasing of the density peaking mainly for the hybrid scenario. Such behaviour will be carefully analysed as it may lead to extra W accumulation in DT. However, this isotope effect obtained could be even an underestimation of the real one. This is because extra effects playing a role on the isotope effect which are not taken into account in the modelling performed (or they are taken into account in a rough way) could reinforce such trend. Electromagnetic effects, which can be isotope dependent, fast ions effects (for instance fast alphas obtained from fusion reactions) and zonal flows are not properly described in the present modelling and however they can play a significant role in DT plasmas [31]. This especially important for ITER DT plasmas, as turbulence for ITER will rely close to threshold, where such effects are stronger. Moreover, the dependence of the pedestal on the isotope is not fully understood yet but there is a clear trend to increase pedestal pressure with average mass [20].

This points out to the necessity of improving the simplified models applied in the framework of integrated modelling, notably the aspects concerning the edge plasma, such as pedestal characteristics and SOL interaction, which are crucial to properly predict DT plasmas self-consistently. However, an effort is also required to close the gap between quasi-linear models and non-linear gyrokinetic simulations in order to capture some non-linear physics, which seems to be essential to account for the physics in advanced scenario regimes. The JET programme is aiming to stablish the framework to perform such activities by providing, in addition, the required experimental data for the validation process. These extra studies and analyses will continue in the following years. 


\section{ACKNOWLEDGEMENTS}

This work has been carried out within the framework of the EUROfusion Consortium and has received funding from the Euratom research and training programme 2014-2018 under grant agreement No 633053. The views and opinions expressed herein do not necessarily reflect those of the European Commission.

\section{REFERENCES}

[1] BELL M.G. et al Nucl. Fusion 35 (1995) 1429

[2] JACQUINOT J. and JET Team, Nucl. Fusion 38 (1998) 1263

[3] GARCIA J. et al Plasma Phys. Control. Fusion 59 (2017) 014023

[4] JOFFRIN E. et al "Overview of the JET preparation for deuterium-tritium operation" 27th IAEA Fusion Energy Conference - 22-27 October 2018 OV1-3

https://conferences.iaea.org/indico/event/151/papers/5940/files/4888-IAEA2018-Joffrin-Overview-paper12p.pdf

[5] STAEBLER G M et al Phys. Plasmas 12 (2005) 102508

[6] BOURDELLE C et al. Plasma Phys. Control. Fusion 58 (2016) 014036

[7] HONDA M. et al. Nucl. Fusion 46 (2006) 580

[8] HOULBERG W.A. et al., Phys. Plasmas 4 (1997) 3230

[9] ARTAUD J.F. et al Nucl. Fusion 50 (2010) 043001

[10] ROMANELLI M et al Plasma Fusion Res. 9 (2014) 3403023

[11] NA Y.S. et al, submitted to Nuclear Fusion

[12] CHALLIS C.D. et al Nucl. Fusion 55 (2015) 053031

[13] MORALES J. et al., EPS 2018 P4.1077

[14] CORDEY J.G. Nucl. Fusion 43 (2003) 670

[15] GARCIA J. et al Nucl. Fusion 54 (2014) 093010

[16] CITRIN J. et al Phys. Rev. Lett. 111 (2013) 155001

[17] GARCIA J. et al Nucl. Fusion 55 (2015) 053007

[18] DUMONT R. J. et al Nucl. Fusion 58 (2018) 082005

[19] HAYASHI N. et al Nucl. Fusion 57 (2017) 126037

[20] MAGGI C. F. et al Plasma Phys. Control. Fusion 60 (2018) 014045

[21] BUSTOS A. et al Physics of Plasmas 22 (2015) 012305

[22] NAKATA M., et al H. Phys. Rev. Lett. 118 (2017) 165002

[23] JENKO F et al Physics of Plasmas 7 (2000) 1904

[24] GARCIA J et al Nucl. Fusion 57 (2017) 014007

[25] WEISEN H. et al. "Isotope Dependence of Confinement in JET-ILW Deuterium and Hydrogen Plasmas" 27th IAEA Fusion Energy Conference 22-27 October 2018 EX/P1-4 https://conferences.iaea.org/indico/event/151/papers/5859/files/5124-IAEA_isotope_8p_corrected.pdf

[26] GALLART D. et al Nucl. Fusion $\mathbf{5 8}$ (2018) 106037

[27] KIROV K.K. et al Nucl. Fusion 59 (2019) 056005

[28] CASSON F.J. et al "Predictive multi-channel flux-driven modelling to optimise ICRH tungsten control in JET" 27th IAEA Fusion Energy Conference - 22-27 October 2018 TH/3-2

https://conferences.iaea.org/indico/event/151/papers/6118/files/4766-Casson_IAEA_2018_4.pdf

[29] BELLI, E. A. and CANDY, J. Plasma Phys. Control. Fusion, 50 (2008) 95010

[30] SAARELMA S. et al Plasma Phys. Control. Fusion 60 (2018) 014042

[31] GARCIA J. et al. Physics of Plasmas 25 (2018) 055902

[32] KAZAKOV Ye.O. et al Nucl. Fusion 55 (2015) 032001

[33] ERIKSSON L.-G. Nucl. Fusion 33 (1993) 1037

[34] CASSON F. J. et al Plasma Phys. Control. Fusion 57 (2015) 014031

[35] NAZIKIAN R. et al Phys. Rev. Lett. 78 (1997) 2976 